Since the partition coefficients were determined at blood $\mathrm{pH}$, the data may be transferred to in vivo conditions. The partition coefficient of caffeine, at least 100 fold higher than that of theophylline and theobromine, explains its rapid penetration into the CSF. The low plasma binding of caffeine and theobromine agrees with the almost complete equilibrium between plasma and CSF concentrations. The somewhat lower relative CSF concentrations of theophylline may not be significant. The comparison of the extracted substances with the injected compounds, investigated by means of thin layer chromatography, showed in case of caffeine, theophylline and theobromine, even several hours after injection, only small traces of substances with $\mathrm{Rf}$ values different from the ones of the injected substances. Thus it is concluded that the measured substance concentration mainly refers to the injected compounds.

Regarding the high partition coefficient of AAT, its small permeation into the CSF is surprising. The chromatographic control of the plasma extract of this substance showed several spots pointing to metabolic products. Nevertheless, the different behaviour of AAT as shown in Figure 1 cannot be explained this way, From the high plasma binding it may be supposed that AAT is bound more intensely by organs than the other purines.
Pilot experiments in rabbits confirmed this supposition. Such an intense binding of AAT by organs would explain its low concentration in the (protein-free) CSF. Thus the quick decline of plasma concentration of AAT can be understood, even though we cannot exclude the biotransformation of AAT into compounds which are not extracted by our method. The behaviour of AAT is especially interesting with regard to its strong central efficiency (HERz et al. ${ }^{3}$ ).

Zusammenfassung. Der Übertritt verschiedener Purinderivate in den Liquor cerebrospinalis nach i.v. Injektion wurde an Hunden untersucht. Coffein permeiert sehr schnell, Theobromin und Theophyllin ungleich langsamer. Dies steht in Einklang mit der unterschiedlichen Lipoidlöslichkeit dieser Substanzen. AAT hingegen tritt nur in geringer Konzentration in den Liquor über, obwohl es die höchste Lipoidlöslichkeit aufweist.

H. J. Teschemacher, A. Herz, R. Hess und G. NovoczeK

Max-Planck-Institut für Psychiatrie, München 23 (Germany), 7 August 1967.

\title{
Fehlen einer cancerogenen Wirkung von nitrosen Gasen beim Goldhamster
}

Von Druckrey und Preussmann ist die Möglichkeit diskutiert worden, die im Tabakrauch enthaltenen nitrosen Gase ( $\mathrm{NO}$ und $\mathrm{NO}_{2}$ ) könnten im Zuge der Inhalation des Rauches mit Gewebsaminen der Schleimhäute des Atemtraktes unter Bildung von cancerogenen Nitrosaminen reagieren ${ }^{1}$. Die langfristige, intermittierende Beatmung von Mäusen mit $40 \mathrm{ppm} \mathrm{NO}_{2}$ erzengt keine bösartigen Tumoren, eine Häufung von gutartigen Lungenadenomen war nicht als cancerogene Wirkung der Stickstoffoxide zu deuten. Wir haben daraus abgeleitet ${ }^{2}$, es bestehe kein Anhalt für eine carcinogene Wirkung nitroser Gase. Diese Folgerung ist dem Einwand begegnet, die Maus stelle nicht das geeignete Versuchstier dar, zum Nachweis cancerogener Effekte am Atemtrakt eigne sich vornehmlich der Goldhamster.

Davon ausgehend, haben wir einen erneuten langfristigen Inhalationsversuch an 40 Goldhamstern durchgeführt. Diese Tierart ist gegenuiber Stickstoffoxiden wesentlich weniger empfindlich als die Maus. Es gelang, die Tiere in $40 \mathrm{ppm} \mathrm{NO}_{2}+20 \mathrm{ppm}$ NO 16 Monate lang praktisch ununterbrochen ohne Todesfolge $z u$ halten. Sie sind lediglich während $1-2 \mathrm{~h}$ täglich zur Sauberhaltung der Käfige an Frischluft verbracht worden. Die Methoden zur Bereitung und analytischen Kontrolle der Stickstoffoxide sind andernorts ${ }^{3}$ beschrieben. Insgesamt standen 36 Hamster für die histologische Auswertung zur Verfügung, von denen 14 zwischen 12 und 16 Monate lang exponiert worden waren. Die Beobachtungszeit betrug $11 / 2$ Jahre. Die Lungen der Goldhamster wurden sofort nach Entnahme in Bouinscher Lösung fixiert und später in Stufenschnitten systematisch und komplett aufgearbeitet, im Regelfall mit Hämatoxylin-Eosin gefärbt.

Bei keinem der Tiere waren maligne Tumoren in den Lungen oder in anderen Organen zu finden. Die Lungenveränderungen entsprechen prinzipiell denen, die wir bereits bei Mäusen in gleichartigen Langzeitversuchen mit $\mathrm{NO}_{2}$ beobachtet haben. Nach Ablauf eines Monats ist hier noch ein massives Lungenödem zu finden, in allen späteren Phasen sieht man mehr oder weniger stark ausgeprägte Zellproliferate, die aus geschwollenen Alveolarepithelien und z.T. auch aus geschwollenen interstitiellen Zellelementen bestehen. Die Prozesse sind durchwegs schwerer als bei Mäusen, vor allem trifft man bei den Hamstern von Anfang an deutlich stärkere entzündliche Reaktionen an Bronchien und Parenchym. In fortgeschrittenen Stadien (nach mehrenen Monaten Begasung) fallen, neben reichlichen Abstossungen von Alveolarepithelien, zahlreiche Mitosen als Hinweis auf regeneratorische Prozesse auf. Man sieht dann oft auch adenomartige Lungenbezirke, in denen Alveolen von zylindrischen Zellen ausgekleidet werden. Die Bronchialepithelien sind manchmal etwas atypisch, jedoch nie in stärkerem Umfang und kaum noch in den zuletzt untersuchten Lungen. Damit ergeben auch Versuche am Goldhamster mit extrem hohen Dosen nitroser Gase, die unausgesetzt einwirken, keinen Anhalt für cine carcinogene Wirkung von Stickstoffoxiden.

Summary. Continuous exposure of golden hamsters to nitrous fumes ( $40 \mathrm{ppm} \mathrm{NO}+20 \mathrm{ppm} \mathrm{NO}$ ) for up to 16 months revealed no carcinogenic action of nitrogen oxides in the lung nor in other organs.

W. Ross und D. Henschler

Pathologisches Institut und Insitut für Toxikologie und Pharmakologie der Universität Wïrzburg (Deutschland), 11. September 1967.

1 H. Druckrey und R. Preussmane, Naturwissenschaften 49, 498 (1962).

2 D. Henschler und W. Ross, Nannyn-Schmiedeberg's Arch. exp. Path. Pharmak. 253, 495 (1966).

- D. Henschler, E. Hahn und W. Assmann, Naunyn-Schmiedeberg's Arch. exp. Path. Pharmak. 249, 325 (1964a). 